\title{
Histological Changes of MaleWestarRats liver Following the Ingestion of Zinc Oxide Nanoparticles With special Emphasis on the Histochemical Alterations
}

Mosaid Abdullah Zaid Alferah*

*Correspondence: frh@qu.edu.sa

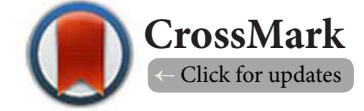

Department of Biology, College of Science and Arts, Unaizah, Qassim University, Saudi Arabia.

\begin{abstract}
The present investigation was carried out on forty apparently healthy mature male Westar rats weighing between 120-200 gm with average three months age. The rats wereobtained from laboratory animal unite in the faculty of pharmacy, King Saud University and were divided randomly into four groups (10 rats/ group). Group I (G1) was kept as a control, Group II (G2)was obtained ZnO NPsin a dose $100 \mathrm{mg} / \mathrm{kg}$ body weight, Group III(G3) was obtained ZnO NPsin a dose $250 \mathrm{mg} / \mathrm{kg}$ BW, Group IV(G4) was obtained $\mathrm{ZnO}$ NPsin a dose $500 \mathrm{mg} / \mathrm{kg} \mathrm{BW}$ and all treated groups received $\mathrm{ZnO}$ NPs by oral gavage for 21 days. G1 showed normal histological structure of all hepatic tissues without any abnormalities. G2 and G3 showed mild to moderate steatosis, necrosis with focal scattered of infammatory cells infltration with fibrous tissue proliferation and moderate hepatocytes glycogen depletion. Meanwhile, G4 showed severe steatosis, diffuse degeneration of hepatic tissues with loss of the hepatic architectures, sever fibrous tissue proliferation with anti-inflammatory cells infiltration as well as severe congestion with the hepatic artery, sinusoids and Portal vein. In addition, sever sinusoidal dilatation accompanied by Kupffer cells hyperplasia in between the hepatic cords were clarified. Furthermore, with PAS stain, sever hepatocytes glycogen depletion were claimed. The present investigation was concluded that the $\mathrm{ZnO}$ NPs have potential oxidative stress in the hepatic tissues that may affect the function of the liver.
\end{abstract}

Keywords: Zinc oxide, nanoparticles, hepatotoxicity, histochemical, ZnO NPs

\section{Introduction}

Nanoparticles (NPs) are materials with a size range of approximately 1-100 $\mathrm{nm}[1]$ that have a very specific chemical and physical properties of size, shape and high ratio of surface area to volume. These qualities have made their suitable application for many medical and biological cases $[2,3]$. And also, Because of their small size, nanoparticles can cross many barriers and filters [4].

Zinc oxide nanoparticles (ZnO NPs) are an inorganic compound with the formula $\mathrm{ZnO}$. It is a white powder that is insoluble in water, and it is widely used as an additive in numerous materials and products including rubbers, plastics, ceramics, glass, cement, lubricants, paints, ointments, adhesives, sealants, pigments, foods, batteries, ferrites, fire retardants, and first-aid tapes. Although it occurs naturally as the mineral zincite, most zinc oxide is produced synthetically. Furthermore, Zinc oxide $\mathrm{ZnO}$ ) nanopowders are available as powders and dispersions and exhibiting antibacterial, anti-corrosive, antifungal and UV filtering properties [5]. Currently, nano-ZnOs are widely used in personal care products; cosmetics and sunscreens [6].

$\mathrm{ZnO}$ NPs are one of the most widely used in consumer products. They are extensively used in cosmetics and sunscreens because of their efficient UV absorption properties. ZnO NPs are being used in the food industry as additives and in packaging due to their antimicrobial properties. They are also being explored for their potential use as fungicides in agriculture and as anticancer drugs and imaging in biomedical applications [7]. In addition, ZnO NPs have important application in the industry of electronic devices and paint industry. Moreover, these particles have been incorporated in polymeric matrices, 
packaging $[8,9]$.

Along with extensive application of $\mathrm{ZnO}$ nanoparticles in the industrial field, it is conceivable that the human body may be intentionally or unintentionally exposed to nanoparticles via several possible routes, including oral ingestion, inhalation, intravenous injection, and dermal penetration. Among these, uptake of nanoparticles by the gastrointestinal tract is one of the most important routes [10].

Ingested nanoparticles may be absorbed through the intestinal lining and translocate into the blood stream where they undergo first pass metabolism in the liver [11]. Biodistribution experiments have revealed liver, kidney and spleen as the target organs for engineered nanoparticles after uptake by the gastrointestinal tract [12]. Other studies indicated potential risks of ZnO NPs on the liver, spleen, lung, kidney and heart as target organs [13,14]. Furthermore, some previous reports showed that $\mathrm{ZnOnanorods}$ were more toxic than spherical ones and the smaller ones were more toxic than the larger ones [15].

Oral administration of $\mathrm{ZnO}$ nanoparticles leads to the release of free $\mathrm{Zn2}+$ ions in gastric acid; thus, $\mathrm{Zn} 2+$ is probably the main toxic material in vivo and can induce cytotoxicity and apoptosis in mammalian cells, [16,17].

$\mathrm{ZnO}$ nanoparticles produce cytotoxic effects in numerous kinds of cells, including osteoblast cancer cells, human bronchial epithelial cells, kidney cells, alveolar adenocarcinoma cells, hepatocytes, and embryonic kidney cells; furthermore, the nanoparticles' effects may be related to particle size and dosage $[18,19]$.

While low doses of $\mathrm{ZnO}$ nanoparticles have not produced toxic effects in vivo, high concentrations can cause sudden death. Moreover, $\mathrm{ZnO}$ nanoparticles are one of the most toxic nanoparticles, with the lowest $\mathrm{LD}_{50}$ (median lethal dose) value, among the engineered metal oxide nanoparticles currently used in research as $\mathrm{TiO}_{2}, \mathrm{Co}_{3} \mathrm{O}_{4^{\prime}} \mathrm{ZnO}$ and $\mathrm{CuO}[20,21]$.

\section{Aim of work}

The present study wasperformed to investigate and evaluate the hepatotoxicity of zinc oxide nanoparticles ofmale Westar rats.

\section{Materials and method \\ Animals and housing}

Forty apparently healthy mature male westar rats weighing between 120-200 gm with average three months age wereobtained from laboratory animal unite in the faculty of pharmacy, King Saud University. The rats were randomly divided into four groups and kept in galvanized standard cages, ten animals/cage, under hygienic conditions and left for one week before starting the experiment for accommodation. Feed and water were available ad libitum. Temperature was recorded continuously, and maintained between (20 and $23^{\circ} \mathrm{C}$ ) along the experimental period. A cycle of $14 \mathrm{~h}$ of light and $10 \mathrm{~h}$ of dark was fixed throughout the experiment. All animals were handled and all experiments were conducted in accordance with the protocols approved by King Saud University Animal Care Ethical Committee while the experimental procedures were carried out in accordance with international guidelines for care and use of laboratory animals.

\section{Supplements (Nanoparticles)}

Well-dispersed ZnO NPs (average particle size 10-30 nm) at $50 \mathrm{wt} \%$ in distilled water (Sigma, Aldrich) were used in the present study. The nanoparticles dispersion had the following characterization: concentration 50 wt.\% in $\mathrm{H} 2 \mathrm{O}$; $\mathrm{pH} 5.5 \pm 0.1$; density $1.7 \mathrm{~g} / \mathrm{ml} \pm 0.1 \mathrm{~g} / \mathrm{ml}$.

\section{Experimental design}

Forty rats were divided randomly into four groups (10 rats/ group) and subjected for 21 days to one of the following treatments:

Group I(G1) kept as a control and fed with a basal diet without ZnO NPs for 21 days.

Group II (G2)was obtained ZnO NPsin a dose 100 mg/kg body weight by oral gavage for 21 days.

Group III(G3)was obtained ZnO NPsin a dose $250 \mathrm{mg} / \mathrm{kg}$ body weight by oral gavage for 21 days.

Group IV(G4)was obtained ZnO NPsin a dose 500 mg/kg body weight by oral gavage for 21 days.

\section{Histological and histochemical processing}

At the end of experiment, cervical dislocation of rats and for histological and histochemical studies, livers were separately and small pieces from them were taken, fixed in neutral buffered formal in $10 \%$, dehydrated, cleared and paraffin ionized for paraffin blocks and 5 micron sections were obtained, mounted on a glass slides and stained with Hematoxylin and Eosin (H\&E), Periodic acid-Schiff (PAS) and Mercuric bromophenol blue according to Bancroft and Gamble [22].

\section{Results}

The histopathological examination of the liver in the control group (G1) revealed normal hepatic architecture; hepatic parenchyma, hepatic lobulation, hepatic cord, portal triad, hepatocytes and hepatic sinusoids. The liver parenchyma of these groups was observed very homogenous, intact and consisting of numerous hepatic lobules that were difficult demarcated from each other's by a very thin connective tissue septa or trabeculae in between, so the hepatic lobulations were not clear (Plate 1: Fig. A). Furthermore, the hepatic lobule appeared hexagonal in shape and had a central vein in their center. The major compartment of each hepatic lobule were the hepatocytes that represent about $80 \%$ of its structure and appeared irregular polygonal or polyhedral shaped cells typically with single, central, large vesicular nucleus with fine dispersed chromatins in most cases, however, some of them appeared occasionally bi-nucleated (Plate 1: Fig. B). 
Hepatocytes were dorsally radiating from the central vein towards the periphery; the portal areas (portal triad) forming the hepatic cords. Moreover, the hepatic sinusoids were observed distributing in between the hepatic cords supplying the hepatocytes with normal, intact lining epithelium (Plate 1: Fig. B). Each hepatic lobule was bounded peripherally with portal triad that housing branches from portal vein, hepatic artery, lymph vessel and bile ductules (Plate 1: Fig. C and D). And also,strongly PAS positive reaction of the hepatic parenchyma was clarified where the glycogen contents were observed filling almost of the hepatocytes cytoplasm (Plate 1: Fig. E and F).

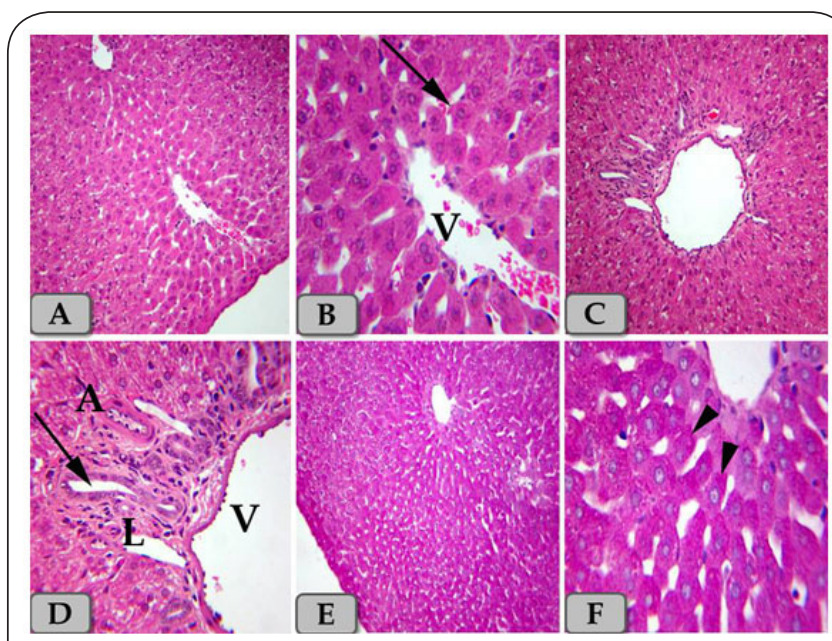

Plate 1. Sections of male Westar rats liver of control group (G1).

Figure (A): showing normal, homogenous, intact hepatic parenchyma; hepatic lobules, with normal central vein. H\&E Obj. x10: Oc.x10. (B): Higher magnification of fig. A showing normal and intact hepatic lobule with normal, regular radiated hepatic cords (arrow) from the central vein (V) to the periphery of lobule. H\&E Obj.x40 : Oc.x10

(C): showing normal and intact portal area. H\&E Obj.x10 : Oc.x10

(D): Higher magnification of fig. C showing intact portal area; portal vein $(\mathrm{V})$, hepatic artery $(\mathbf{A})$, lymph vessel $(\mathrm{L})$ and bile ductules (arrow). H\&E Obj.x20 : Oc.x10.

(E): showing strongly PAS positive reaction of the hepatocytes. PAS Obj.x10 : Oc.x10.

(F): Higher magnification of fig. E showing the glycogen contents that filling almost of the hepatocytes cytoplasm. PAS Obj.x40 : Oc.x10.

Mean while, G2 treated with ZnO NPs in a dose of $100 \mathrm{mg} /$ kg. bwt and G3 treated with ZnO NPs in a dose of $250 \mathrm{mg} /$ $\mathrm{kg}$. bwt showed mild to moderate disorganization of the hepatic cords with moderate degeneration and necrosis of the hepatocytes (Plate 2: Fig. A). Microvesicular steatosis; accumulations of small lipid droplets within the hepatocytes cytoplasm were also observed (Plate 2: Fig. B and C). In addition, focal scattered of inflammatory cells infiltration with

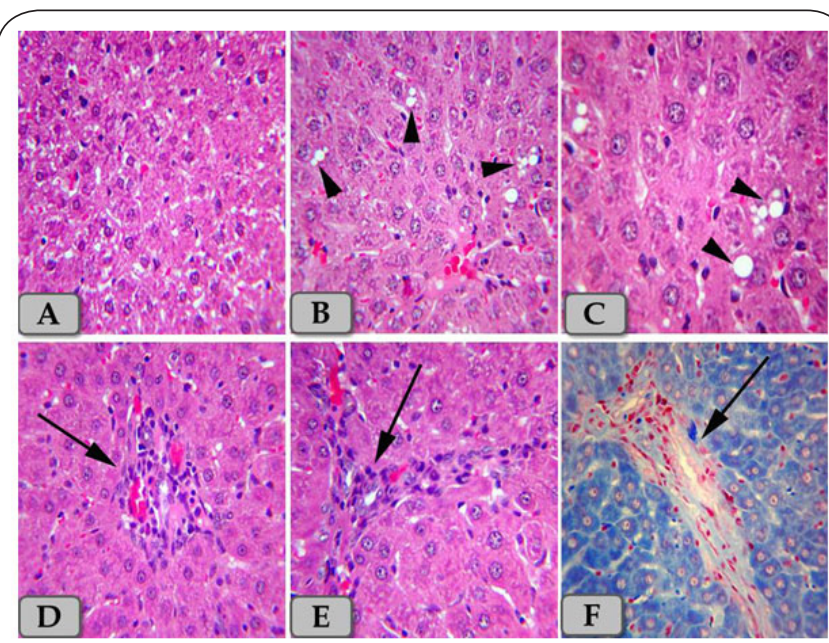

Plate 2. Sections of male Westar ratsliver of G2 treated with $\mathrm{ZnO}$ NPs in a dose of $100 \mathrm{mg} / \mathrm{kg}$. bwt and G3 treated with $\mathrm{ZnO}$ NPs in a dose of $250 \mathrm{mg} / \mathrm{kg}$. bwt by oral gavage for 21 days.

(A): showing moderate disorganization of the hepatic cords with moderate necrosis within the hepatocytes. H\&E Obj.x20 : Oc.x10

(B): showing moderate microvesicularsteatosis; accumulation of small lipid droplets in hepatocytes cytoplasm (arrow head). H\&E Obj.x20 : Oc.x10.

(C): Higher magnification of fig. B showing the microvesicularsteatosis (arrow head). H\&E Obj.x40 : Oc.x10. $(\mathbf{D}, \mathbf{E}$ and $\mathbf{F})$ : showing moderate inflammatory cells infiltration with fibrous connective tissue proliferation within the hepatic parenchyma (arrow). D, E) H\&E. Obj.x20 : Oc.x10 F) Bromo phenol blue Obj.x20 : Oc.x10.

fibrous connective tissue proliferation was demonstrated within the hepatic tissues especially portal areas (Plate 2: Fig. D, E and F). And also, moderate congestion within the portal vein in the portal triad was observed (Plate 3: Fig. G and H). Furthermore, moderate sinusoidal dilatation and congestion in between the hepatic cords were clarified (Plate 3: Fig. I). Moreover, with PAS stain, pale scattered patches of glycogen depletion within the hepatic parenchyma were demonstrated (Plate 3: Fig. J, $\mathrm{K}$ and L).

On the other hand, liver of G4 treated with ZnO NPs in a dose of $500 \mathrm{mg} / \mathrm{kg}$. bwt showed severe microvesicularsteatosis; accumulation of small lipid droplets in hepatocytes cytoplasm (Plate 4: Fig. B and C). And also, diffuse degeneration and necrosis of hepatic tissues with loss of the hepatic architectures were clearly observed (Plate 4: Fig. A). Sever fibrous tissue proliferation with anti-inflammatory cells infiltration; plasma cells, mast cells, kuppfer cells, lymphocytes and eosinophils were observed within the hepatic parenchyma especially portal triad (Plate 4: Fig. D and E). Hexagonal lobules are centered on the central vein that exhibited sever congestion and also within the portal triad, the portal vein was also showed sever congestion that overfilled with erythrocytes and some lymphocytes (Plate 4: Fig. F and Plate 5: Fig. G, H and I). 


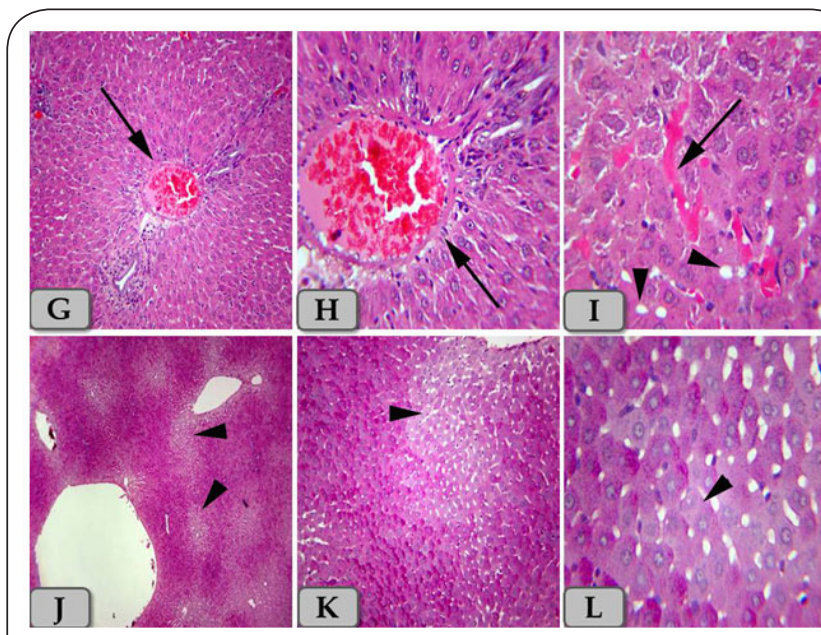

Plate 3. Sections of male Westar ratsliver of G2 and G3. (G): showing moderate congestion within the portal vein in the portal triad (arrow). H\&E Obj.x10 : Oc.x10.

(H): Higher magnification of fig. G showing the same (arrow). H\&E Obj.x20 : Oc.x10

(I): showing the dilatation and congestion within the hepatic sinusoids (arrow) and steatosis (arrow head). H\&E Obj.x40 : Oc.x10.

( $\mathbf{J}, \mathbf{K}$ and $\mathbf{L}$ ): showing scattered pale patches of glycogen depletion within the hepatic parenchyma (arrow head). J, K and L) PAS J) Obj.x4 : Oc.x10 K) Obj.x10 : Oc.x10 L) Obj.x40 : Oc.x10.

Furthermore, disorganization of hepatic cords was observed. In addition, sever degenerative changes which were evident in numerous hepatocytes; enlarged cells, had light and foamy cytoplasm filled with vacuoles of variable size that were tended to form cystic degeneration were claimed. Hepatocytes necrotic changes were evident; a small, pyknotic cellular nuclei with condensed chromatin, lack of nucleolus and acidophilic cytoplasm were recognized (Plate 4: Fig. A and C). Moreover, sever sinusoidal dilatation with congestion accompanied by Kupffer cells hyperplasia in between the hepatic cords were clarified (Plate 5: Fig. I). And also, with PAS stain, pale hepatic parenchyma with sever glycogen depletion within the hepatocytes were noticed (Plate 5: Fig. J, K and L).

\section{Discussions}

The present investigation revealed that the liver of the control group (G1) revealed normal hepatic architecture; hepatic parenchyma, hepatic lobulation, hepatic cord, hepatic portal triad, hepatocytes and hepatic sinusoids. Meanwhile, G2 treated with ZnO NPs in a dose of $100 \mathrm{mg} / \mathrm{kg}$. bwt and G3 treated with ZnO NPs in a dose of $250 \mathrm{mg} / \mathrm{kg}$. bwt showed mild to moderate steatosis and necrosis with moderate disorganization of hepatic cords. In addition, focal scattered of inflammatory cells infiltration with fibrous connective tissue proliferation was demonstrated within the hepatic tissues especially portal areas. Furthermore, moderate sinusoidal

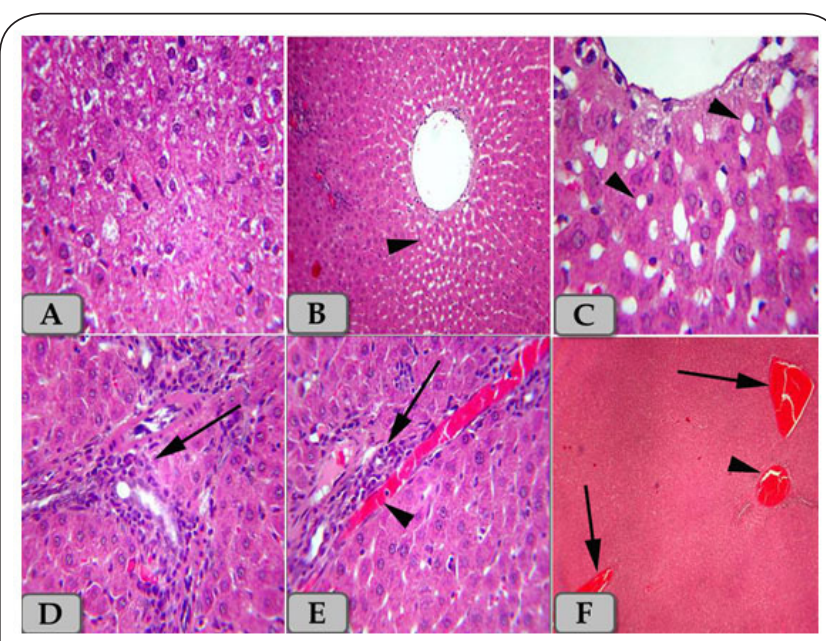

Plate 4. Sections of male Westar ratsliver of G4 treated with $\mathrm{ZnO}$ NPs in a dose of $500 \mathrm{mg} / \mathrm{kg}$. bwt by oral gavage for 21 days.

(A): showing sever disorganization of the hepatic cords, severe necrosis in the hepatocytes with pyknotic cellular nuclei and condensed chromatin. H\&E Obj.x20 : Oc.x10.

(B and C): showing severmicrovesicularsteatosis (arrow head). C) Higher magnification of fig. B showing the same. B, C) H\&E B) Obj.x10 : Oc.x10 C) Obj.x40 : Oc.x10.

(D and $\mathbf{E})$ :showing sever inflammatory cells infiltration with sever fibrous connective tissue proliferation within the hepatic parenchyma (arrow) with sever dilatation and congestion within the hepatic sinusoids (arrow head). D, E) H\&E Obj. $\mathrm{x} 20$ : Oc.x10.

(F):showing sever congestions within the portal vein in the portal triad (arrow head) and central vein (arrow). H\&E Obj.x4 : Oc.x10.

dilatation in between the hepatic cords and hepatocytes glycogen depletion were also observed.

With increasing dose of $\mathrm{ZnO} N \mathrm{Ns}$, the liver toxicity became more obvious where, the liver of G4 treated with ZnO NPs in a dose of $500 \mathrm{mg} / \mathrm{kg}$. bwt showed severe microvesicularsteatosis; accumulation of small lipid droplets in hepatocytes cytoplasm.Such finding is completely goes hand in hand withthe description of Khorsandi etal., [23] who clarified that lobular structures of the liver were damaged in NZnO-intoxicated rats. Centrilobular hepatocytes showed microvesicularsteatosis, accumulation of erythrocytes in central vein and sinusoids and infltration of infammatory cells were also observed.

The present study illustrated thatdiffuse degeneration and necrosis of hepatic tissues with loss of the hepatic architectures were clearly observed. This results is in parallelism with Singh et al., [24] who described that ZnO NPs induced hepatocytes necrosis and this finding may indicate recent hepatotoxicity injury resulted from the cessation of protein synthesis due to subjection to these nanoparticles. Necrosis is induced by toxicants that attack the cell organelles specially the mitochondria, endoplasmic reticulum and nucleus thus disturbing their activity. 


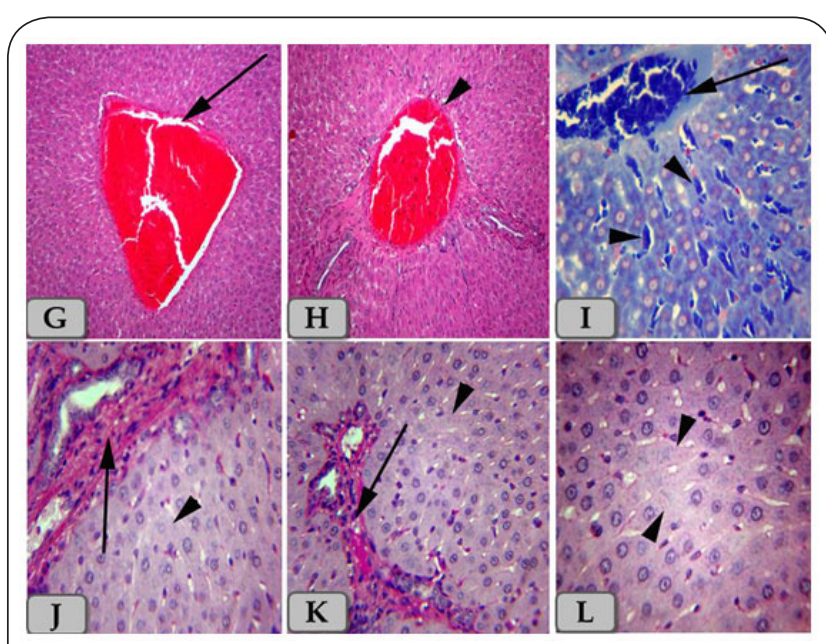

Plate 5. Sections of male Westar ratsliver of G4

(G): Higher magnification of fig. F showing sever congestion within the central vein (arrow). H\&E Obj.x10 : Oc.x10.

$(\mathbf{H})$ : Higher magnification of fig. F showing sever congestion within the portal vein in the portal triad (arrow head). H\&E Obj.x10 : Oc.x10.

(I): showing sever congestion within the central vein (arrow) and sever congestion within the hepatic sinusoids (arrow head). Bromo phenol blue Obj.x20: Oc.x10. ( $\mathbf{J}, \mathbf{K}$ and $\mathbf{L}$ ): showing pale hepatic parenchyma with sever glycogen depletion within the hepatic parenchyma (arrow head) and sever inflammatory cell infiltrations with fibrous tissue proliferation (arrow). J, K and L) PAS J, K) Obj.x20 : Oc.x10 L) Obj.x40 : Oc.x10.

Sever fibrous tissue proliferation with anti-inflammatory cells infiltration; plasma cells, mast cells, kuppfer cells, lymphocytes and eosinophils were observed within the hepatic parenchyma especially portal triad. Hexagonal lobules are centered on the central vein that exhibited moderate to severe congestion with the hepatic artery, sinusoids and Portal vein. These investigations are coinciding with Landsiedel et al., [25] and Watson et al., [26] who claimed that the histological analysis of liver sections showed areas of necrosis accompanied by significant inflammatory cell infiltration near the hepatic portal triad at day 7 of exposure. By this time, the engulfed ZnO ENPs would have dissolved, releasing zinc ions, which may have caused toxicity to the surrounding hepatocytes.

Furthermore, disorganization of hepatic cords was observed. In addition, sever degenerative changes which were evident in numerous hepatocytes; enlarged cells, had light and foamy cytoplasm filled with vacuoles of variable size that were tended to form cystic degeneration were claimed. Hepatocytes necrotic changes were evident; a small, pyknotic cellular nuclei with condensed chromatin, lack of nucleolus and acidophilic cytoplasm were observed were recognized. These results are in parallelism with the result of Almansour et al., [27] who observed that the liver of ZnO NPs treated rats for 21 days demonstrated well-defined necrotic hepatocytes. And also, hepatocytes cyto- plasmic vacuolation with partial cytoplasmic swelling was well demonstrated. Moreover, several forms of nuclear abnormality were exhibited;binucleation, nuclear vesiculation, anisokaryosis, karyolysis and nuclear membrane irregularity and apopotosis.

Sever sinusoidal dilatation accompanied by Kupffer cells hyperplasia in between the hepatic cords were clarified. These findings are in agreement with the results of Almansour et al., [27] who showed that the liver of ZnO NPs treated rats exhibited sinusoidal dilatation accompanied by Kupffer cells activation and enlarged that lining the walls of sinusoids. This abnormality was also demonstrated in the toluidine blue stained semi thin sections. This vascular alteration was characterized by widening of capillaries lining the hepatic strands.Moreover, our findings are also goes hand in hand with Oligny and Lough [28] and Neyrinck [29] who reported that the sinusoidal dilatation in the liver of rats treated with ZnO NPs might be resulted from an injury of their sinusoids endothelia. On the other hand, Kupffer cells hyperplasia might be a sort of defense mechanism of detoxification contributedto hepatic oxidative stress induced by these particles.

Our investigation of Kupffer cells activation and hyperplasia in between the hepatic cords were supported by the description of Hanley et al., [30] who clarified that Zinc oxide NPs were affect monocytes and macrophages by initiating production of interferon tumor necrosis factor by the peripheral blood monocytes.

With PAS stain, sever hepatocytes glycogen depletion were observed. These investigations are in coincidence with the description of Almansour et al., [27] who observed that PAS stain exhibited partial hepatocytes glycogen content depletion. The later, was mainly observed in the degenerative hepatocytes. Moreover, Prussian blue reaction demonstrated precipitation of hemosiderin pigments in the hepatic tissues of $\mathrm{ZnO}$ NPs treated group.

The detected apoptosis in the liver of rats treated with $\mathrm{ZnO}$ NPs might be resulted from intercellular stress induced by these fine particles [31]. Apoptosis might be followed by mitochondrial swelling, endoplasmic reticulum dilatation and lysosomal rupture before shrinking and dissolution of nuclei [32].

\section{Conclusion}

From our results, we can conclude that the $\mathrm{ZnO}$ NPs have potential oxidative stress in the hepatic tissues that may affect the function of the liver.

\section{Competing interests}

The author declares that he has no competing interests.

\section{Acknowledgements}

I wish to express my deepest gratitude and sincere thanks to: 1) Dr. Wael A.M. Ghonimi, Professor Assistant of Histology and Cytology, Faculty of Veterinary Medicine, Zagazig University, Zagazig, Egypt for hishelp, support and efforts in the histological examination, images capture, result reading and language editing. 2) Mr. Tayseir Rashed Almunizel, laboratory technician at the Faculty of Pharmacy, King Saud University, Riyadh, Saudi Arabia 
who contributed to the care of animals during the experiment 3) Mr. Khalid Elfaki Ibrahim, technician at the Central Laboratory, Department of Zoology, King Saud University, Riyadh, Saudi Arabia who contributed to the histological cutting and staining of sections.

4) Qassim University, for its financial support for this research.

Publication history

EIC: Gaetano Giuseppe Magro, University of Catania, Italy.

Received: 24-Dec-2017 Final Revised: 12-Jan-2018

Accepted: 18-Jan-2018 Published: 27-Jan-2018

\section{References}

1. Geneva, Switzerland. Nanotechnologies-Terminology and Definitions for Nano-objects-Nanoparticle, Nanofibre and Nanoplate. International Organization for Standardization. 2008; 32:1-7.

2. Erb U, Aust KT and Palumbo G. In nanostructured materials. processing, properties and potential applications. Noyes: New York. 2002; 179-222.

3. Roduner E. Size matters: why nanomaterials are different. Chem Soc Rev. 2006; 35:583-92. | Article | PubMed

4. Shirvani $H$, Noori A and Mashayekh AM. The Effect of ZnO Nanoparticles on the Growth and Puberty of Newborn Male Wistar Rats. International Journal of Basic Sciences \& Applied Research. 2014; 3:180-185. | Pdf

5. Liedekerke M. De. "2.3. Zinc Oxide (Zinc White): Pigments, Inorganic, 1" In Ullmann's Encyclopedia of Industrial Chemistry. Weinheim: Wiley-VCH. 2006.

6. Ma H, Williams PL and Diamond SA. Ecotoxicity of manufactured ZnO nanoparticles--a review. Environ Pollut. 2013; 172:76-85. | Article | PubMed

7. Rasmussen JW, Martinez E, Louka P and Wingett DG. Zinc oxide nanoparticles for selective destruction of tumor cells and potential for drug delivery applications. Expert Opin Drug Deliv. 2010; 7:1063-77. Article | PubMed Abstract | PubMed FullText

8. Vandebriel RJ and De Jong WH. A review of mammalian toxicity of $\mathrm{ZnO}$ nanoparticles. Nanotechnol Sci Appl. 2012; 5:61-71. | Article | PubMed Abstract | PubMed FullText

9. Tayel A, El-Tras W, Moussa S, El-Baz A, Maahrous H, Salem M and Brimer $L$. Antibacterial action of zinc oxide nanoparticles against food borne pathogens. J. Food Saf. 2011; 31:211-218.

10. Saman S, Moradhaseli S, Shokouhian A and Ghorbani M. Histopathological effects of $\mathrm{ZnO}$ nanoparticles on liver and heart tissues in wistar rats. Adv. Biores. 2013; 2:83-88.

11. Bockmann J, Lahl H, Eckert T and Unterhalt B. [Titanium blood levels of dialysis patients compared to healthy volunteers]. Pharmazie. 2000; 55:468. | PubMed

12. Cui Y, Liu H, Zhou M, Duan Y, Li N, Gong X, Hu R, Hong M and Hong F. Signaling pathway of inflammatory responses in the mouse liver caused by TiO2 nanoparticles. J Biomed Mater Res A. 2011; 96:221-9. | Article | PubMed

13. Jachak A, Lai SK, Hida K, Suk JS, Markovic N, Biswal S, Breysse PN and Hanes J. Transport of metal oxide nanoparticles and single-walled carbon nanotubes in human mucus. Nanotoxicology. 2012; 6:614-22. | Article | PubMed Abstract | PubMed FullText

14. Li CH, Shen CC, Cheng YW, Huang SH, Wu CC, Kao CC, Liao JW and Kang JJ. Organ biodistribution, clearance, and genotoxicity of orally administered zinc oxide nanoparticles in mice. Nanotoxicology. 2012; 6:746-56. | Article | PubMed

15. Hsiao I and Huang Y. Effects of various physioche- mical Characteristics on the toxicities of $\mathrm{ZnO}$ and $\mathrm{TiO} 2$ nanoparticles toward Human lung epithelial cell. Sci. Total Environ. 2011; 409:1219-1228.

16. Gojova A, Guo B, Kota RS, Rutledge JC, Kennedy IM and Barakat AI. Induction of inflammation in vascular endothelial cells by metal oxide nanoparticles: effect of particle composition. Environ Health Perspect.
2007; 115:403-9. | Article | PubMed Abstract | PubMed FullText

17. Jeng $\mathrm{HA}$ and Swanson J. Toxicity of metal oxide nanoparticles in mammalian cells. J Environ Sci Health A Tox Hazard Subst Environ Eng. 2006; 41:2699-711. | Article | PubMed

18. Nair S, Sasidharan A, Divya Rani VV, Menon D, Manzoor K and Raina S. Role of size scale of $\mathrm{ZnO}$ nanoparticles and microparticles on toxicity toward bacteria and osteoblast cancer cells. J Mater Sci Mater Med. 2009; 20 Suppl 1:S235-41. | Article | PubMed

19. Kang $T$, Guan $R$, Chen $X$, Song $Y$, Jiang $H$ and Zhao J. In vitro toxicity of different-sized ZnO nanoparticles in Caco-2 cells. Nanoscale Res Lett. 2013; 8:496. | Article | PubMed Abstract | PubMed FullText

20. De Angelis I, Barone F, Zijno A, Bizzarri L, Russo MT, Pozzi R, Franchini F, Giudetti G, Uboldi C, Ponti J, Rossi F and De Berardis B. Comparative study of $\mathrm{ZnO}$ and $\mathrm{TiO}(2)$ nanoparticles: physicochemical characterisation and toxicological effects on human colon carcinoma cells. Nanotoxicology. 2013; 7:1361-72. | Article | PubMed

21. Yin $\mathrm{H}$, Casey PS, McCall MJ and Fenech M. Effects of surface chemistry on cytotoxicity, genotoxicity, and the generation of reactive oxygen species induced by ZnO nanoparticles. Langmuir. 2010; 26:15399408. | Article | PubMed

22. Bancroft JD and Gamble M. Theory and practice of histological techniques, 5th Ed. Churchill livingstone. 2001; 281-285.

23. Khorsandi L, Mansouri E, Orazizadeh M and Jozi Z. Curcumin Attenuates Hepatotoxicity Induced by Zinc Oxide Nanoparticles in Rats. Balkan Med J. 2016; 33:252-7. | Article | PubMed Abstract | PubMed FullText

24. Singh $A$, Bhat $T$ and Sharma O. Clinical biochemistry and hepatotoxicity. J.Clin. Toxicol. 2011; S4:001.

25. Landsiedel R, Ma-Hock L, Van Ravenzwaay B and Oesch F. Gen toxicitystudies on titanium dioxide and zinc oxide nanomaterials used forUV-protection in cosmetic formulation. Nanotoxicology. 2010; 4:364-381.

26. Watson CY, Molina RM, Louzada A, Murdaugh KM, Donaghey TC and Brain JD. Effects of zinc oxide nanoparticles on Kupffer cell phagosomal motility, bacterial clearance, and liver function. Int J Nanomedicine. 2015; 10:4173-84. | Article | PubMed Abstract | PubMed FullText

27. Almansour MI, Alferah MA, Shraideh ZA and Jarrar BM. Zinc oxide nanoparticles hepatotoxicity: Histological and histochemical study. Environ Toxicol Pharmacol. 2017; 51:124-130. | Article | PubMed

28. Oligny LL and Lough J. Hepatic sinusoidal ectasia. Hum Pathol. 1992; 23:953-6. | PubMed

29. Neyrinck A. Modulation of Kupffer cell activity: physio-pathological consequences on hepatic metabolism. Bull Mem Acad R Med Belg. 2004; 159:358-66. | PubMed

30. Hanley C, Thurber A, Hanna C, Punnoose A, Zhang J and Wingett DG. The Influences of Cell Type and ZnO Nanoparticle Size on Immune Cell Cytotoxicity and Cytokine Induction. Nanoscale Res Lett. 2009; 4:140920. | Article | PubMed Abstract | PubMed FullText

31. Meyer K, Rajanahalli P, Ahamed M, Rowe JJ and Hong Y. ZnO nanoparticles induce apoptosis in human dermal fibroblasts via p53 and p38 pathways. Toxicol In Vitro. 2011; 25:1721-6. | Article | PubMed

32. Johar D, Roth JC, Bay GH, Walker JN, Kroczak TJ and Los M. Inflammatory response, reactive oxygen species, programmed (necrotic-like and apoptotic) cell death and cancer. Rocz Akad Med Bialymst. 2004; 49:31-9. | PubMed

\section{Citation:}

Alferah MAZ. Histological Changes of MaleWestarRats liver Following the Ingestion of Zinc Oxide Nanoparticles With special Emphasis on the Histochemical Alterations. J Histol Histopathol. 2018; 5:4. http://dx.doi.org/10.7243/2055-091X-5-4 